\title{
The restricted Inomata-McKinley spinor- plane, homotopic deformations and the Lounesto classification
}

Cite as: J. Math. Phys. 60, 042301 (2019); https://doi.org/10.1063/1.5086440

Submitted: 20 December 2018 . Accepted: 12 March 2019 . Published Online: 05 April 2019

D. Beghetto, R. J. Bueno Rogerio, and C. H. Coronado Villalobos
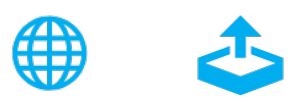

\section{ARTICLES YOU MAY BE INTERESTED IN}

The Gaussian integration method of the Schrödinger equation and quantum 1-D theory of low gain free electron laser

Journal of Mathematical Physics 60, 042101 (2019); https://doi.org/10.1063/1.5040925

On the spectrum-generating superalgebras of the deformed one-dimensional quantum oscillators

Journal of Mathematical Physics 60, 042102 (2019); https://doi.org/10.1063/1.5085164

The stability of free fermi Hamiltonians

Journal of Mathematical Physics 60, 042201 (2019); https://doi.org/10.1063/1.5053869

Where in the world is AIP Publishing?

Find out where we are exhibiting next 


\title{
The restricted Inomata-McKinley spinor-plane, homotopic deformations and the Lounesto classification
}

\author{
Cite as: J. Math. Phys. 60, 042301 (2019); doi: 10.1063/1.5086440 \\ Submitted: 20 December 2018 - Accepted: 12 March 2019 • \\ Published Online: 5 April 2019
}

\author{
D. Beghetto, ${ }^{1, a)}$ R. J. Bueno Rogerio, ${ }^{1,2, b)}$ and C. H. Coronado Villalobos ${ }^{3,(,)}$
}

\begin{abstract}
AFFILIATIONS
${ }^{1}$ Departamento de Física e Química, Faculdade de Engenharia de Guaratinguetá, Universidade Estadual Paulista (UNESP), 12516-410 Guaratinguetá, SP, Brazil

${ }^{2}$ Instituto de Física e Química, Universidade Federal de Itajubá-IFQ/UNIFEI, Av. BPS 1303, CEP 37500-903 Itajubá, MG, Brazil

${ }^{3}$ Instituto Nacional de Pesquisas Espaciais (INPE) Ciências Espaciais e Atmosféricas 12227-010 São José dos Campos, SP, Brazil
\end{abstract}

a) Electronic mail: dbeghetto@feg.unesp.br

b) Electronic mail: rodolforogerio@unifei.edu.br

${ }^{c}$ Electronic mail: ccoronado@id.uff.br

\begin{abstract}
We define a two-dimensional space called the spinor-plane, where all spinors that can be decomposed in terms of Restricted InomataMcKinley (RIM) spinors reside, and describe some of its properties. Some interesting results concerning the construction of RIMdecomposable spinors emerge when we look at them by means of their spinor-plane representations. We show that, in particular, this space accommodates a bijective linear map between mass-dimension-one and Dirac spinor fields. As a highlight result, the spinor-plane enables us to construct homotopic equivalence relations, revealing a new point of view that can help us to give one more step toward the understanding of the spinor theory. In the end, we develop a simple method that provides the categorization of RIM-decomposable spinors in the Lounesto classification, working by means of spinor-plane coordinates, which avoids the often hard work of analyzing the bilinear covariant structures one by one.
\end{abstract}

\section{INTRODUCTION}

The so-called Inomata-McKinley spinors are a particular class of solutions of the non-linear Heisenberg equation. ${ }^{1}$ A subclass of Inomata-McKinley spinors called Restricted Inomata-McKinley (RIM) spinors was revealed to be useful in describing neutrino physics. $^{2}$ It is well known that free linear massive (or mass-less) Dirac fields can be represented as a combination of RIM-spinors. ${ }^{2}$ Moreover, it was recently shown ${ }^{3}$ that such Dirac spinors are necessarily type-1 in the so-called "Lounesto classification" and that they are all non-exotic spinors: i.e., the spacetime itself needs to have an underlying trivial topology ${ }^{28}$ in order to enable the very existence of RIM-spinors. Thus, the decomposition in terms of RIM-spinors itself is not allowed in a spacetime with non-trivial topology.

The Elko eigenspinors of charge conjugation operator, which are mass-dimension-one (MDO) spinors (we will use "MDO" to call these spinor fields), compose a new set of spinors with an interesting and complex structure on its own. ${ }^{4,5}$ MDO spinors form a complete set of eigenspinors of the charge conjugation operator, $C$; however, they have dual helicity and can take positive (self-conjugated) and negative (anti-self-conjugated) eigenvalues of $C$, contrasting with the Majorana, which take only the positive value and carry singlehelicity. From the physical point of view, such spinors are constructed to be "invisible" to other particles, once all the couplings with the fields of the Standard Model (SM) are not allowed, except for the Higgs boson, thus becoming a natural candidate to describe dark matter. 
The idea of mapping MDO and Dirac spinor fields is not new. ${ }^{6-8}$ However, the works developed toward this proposal use MDO as being a type- 5 spinor field within Lounesto classification, taking the bilinear covariants associated with this class as fundamental elements in the construction of the mapping. It is well known that MDO fields do not fulfill the requisites to fit in the Lounesto classification (for more details, the reader is referred to Ref. 9), since their dual is defined in a different way than the one usually imposed in such a classification. Moreover, MDO fields are governed by a whole non-usual dynamics, carrying a new and different physical content. Then, a true map between Dirac and MDO spinors is, in fact, a map between different spinor spaces. Thus, to transcend the need of using the bilinear structures associated with the spinors would be welcoming in such an attempt to construct the aforementioned map.

Topology is a very important field of study not only in Mathematics but also in many areas of Physics. By means of its methods and concepts, topology often allows the discovery and a deep understanding of several substantial aspects in condensed matter, cosmology, and many other fields. Furthermore, in particular, by homotopical tools, interesting connections between only apparently disconnected areas and results are often revealed, which makes this field of study very powerful and interesting.

In the present work, we construct a space called spinor-plane, which is a two-dimensional space with its elements being every spinor that can be written in terms of RIM-spinors. The study of this space leads to a better understanding of properties and relations between these spinors, as we shall see. The fundamental concept, in Algebraic Topology, of homotopic maps reveals to be impressive in the study of the spinors in this plane. Moreover, by means of the spinor-plane, we provide a truthful and direct categorization of RIMdecomposable spinors in the so-called Lounesto classification of spinor fields. Also, we show that an easily constructible bijective map between Dirac and MDO spinors is a direct result of the properties of the spinor-plane, dealing only with their decompositions in terms of RIM-spinors.

This paper is organized as follows: A short elementary review on the Lounesto classification and on RIM-spinors is presented in two separated subsections in Sec. II. The decomposition of MDO spinors in terms of RIM-spinors is made in Sec. III. In Sec. IV, we construct the two-dimensional space of all RIM-decomposable spinors and present some of its properties, with the main results being shown as two lemmas. Strong results relating homotopy and RIM-decomposable spinors are condensed in two theorems rigorously constructed in Sec. V. In Sec. VI, we devote our attention to the bilinear covariants of the particular class of RIM-decomposable spinors that has its adjoint defined in the Dirac fashion, with the results presented as two propositions. In Sec. VII, we conclude.

\section{ELEMENTARY REVIEW}

This section is reserved for a small review on the introductory elements that are necessary for the study carried out in the scope of this paper.

\section{A. The Lounesto classification}

Let $\psi$ be an arbitrary spinor field, belonging to a section of the vector bundle $\mathbf{P}_{\operatorname{spin}_{1,3}^{e}}(\mathcal{M}) \times{ }_{\rho} \mathbb{C}^{4}$, where $\rho$ stands for the entire representation space $D^{(1 / 2,0)} \oplus D^{(0,1 / 2)}$. The usual bilinear covariants associated with $\psi$ reads

$$
\begin{aligned}
A & =\bar{\psi} \psi \text { (scalar), } \\
B & =i \bar{\psi} \gamma_{5} \psi \text { (pseudo-scalar), } \\
\mathbf{J} & =J_{\mu} \theta^{\mu}=\bar{\psi} \gamma_{\mu} \psi \theta^{\mu} \text { (vector), } \\
\mathbf{K} & =K_{\mu} \theta^{\mu}=\bar{\psi} i \gamma_{0123} \gamma_{\mu} \psi \theta^{\mu} \text { (axial-vector), } \\
\mathbf{S} & =S_{\mu \nu} \theta^{\mu \nu}=\frac{1}{2} \bar{\psi} i \gamma_{\mu \nu} \psi \theta^{\mu} \wedge \theta^{v} \text { (bi-vector), }
\end{aligned}
$$

where $\gamma_{0123}:=\gamma_{5}=\gamma_{0} \gamma_{1} \gamma_{2} \gamma_{3}$ and $\gamma_{\mu \nu}:=\gamma_{\mu} \gamma_{\nu}$. Denoting by $\eta_{\mu \nu}$ the Minkowski metric, the set $\left\{\mathbb{1}, \gamma_{I}\right\}$ (where $I \in\{\mu, \mu \nu, \mu \nu \rho, 5\}$ is a composed index) is a basis for the Minkowski spacetime $\mathcal{M}(4, \mathbb{C})$ satisfying $\gamma_{\mu} \gamma_{v}+\gamma_{v} \gamma_{\mu}=2 \eta_{\mu v} \mathbb{1}$, and $\bar{\psi}=\psi^{\dagger} \gamma_{0}$ stands for the adjoint spinor with respect to the Dirac dual. Yet, the elements $\left\{\theta^{\mu}\right\}$ are the dual basis of a given inertial frame $\left\{\mathbf{e}_{\mu}\right\}=\left\{\frac{\partial}{\partial x^{\mu}}\right\}$, with $\left\{x^{\mu}\right\}$ being the global spacetime coordinates. Also, we denote $\theta^{\mu v}:=\theta^{\mu} \wedge \theta^{v}$.

In the Dirac theory, the above bilinear covariants are interpreted respectively as the mass of the particle $(\sigma)$, the pseudo-scalar $(\omega)$ relevant for parity-coupling, the current of probability $(\mathbf{J})$, the direction of the electron spin $(\mathbf{K})$, and the probability density of the intrinsic electromagnetic moment (S) associated with the electron. In general grounds, it is always expected to associate such bilinear structures with physical observables.

The bilinear forms defined in (1)-(5) obey the so-called Fierz-Pauli-Kofink (FPK) identities given by ${ }^{10}$

$$
\begin{aligned}
J^{2} & =A^{2}+B^{2}, \\
J_{\mu} K_{v}-K_{\mu} J_{v} & =-B S_{\mu v}-\frac{A}{2} \epsilon_{\mu \nu \alpha \beta} S^{\alpha \beta},
\end{aligned}
$$




$$
\begin{aligned}
J_{\mu} K^{\mu} & =0, \\
J^{2} & =-\boldsymbol{K}^{2} .
\end{aligned}
$$

So, the algebraic constraints presented in (1)-(5) reduce the possibilities of (only) six different spinor classes (for which $\boldsymbol{J}$ is always non-null), known as Lounesto classification,

$$
\begin{array}{lll}
\text { 1. } & A=0, \quad B \neq 0 ; & \\
\text { 2. } & A=0, \quad B=0 ; & \\
\text { 3. } & A=0, \quad B \neq 0 ; \\
\text { 4. } & A=0=B, \quad \mathbf{K} \neq 0, & \mathbf{S} \neq 0 ; \\
\text { 5. } & A=0=B, \quad \mathbf{K}=0, & \mathbf{S} \neq 0 ; \\
\text { 6. } & A=0=B, \quad \mathbf{K} \neq 0, & \mathbf{S}=0,
\end{array}
$$

with classes 1,2 , and 3 satisfying $\mathbf{K}, \mathbf{S} \neq 0$. The spinors belonging to the first three classes are called regular spinors, while classes 4 , 5 , and 6 are labeled singular spinors. ${ }^{4,8,12}$ Spinors describing fermions in field theory are called Dirac spinors, and they may belong to classes 1, 2, or 3; i.e., all Dirac spinors are necessarily regular ones (but not all regular spinors are necessarily Dirac spinors, as shown in Ref. 15).

As was recently shown in Ref. 9, due to the adjoint structure of the MDO fermions, ${ }^{5}$ it is extremely necessary to deform the usual Clifford algebra in order to ascertain the right observance of the FPK identities, regarding MDO spinor fields.

\section{B. A short overview on the non-linear Heisenberg theory formalism} by $^{16-18}$

The non-linear Heisenberg equation of motion is easily obtained by varying the action with respect to the spinor field, constructed

$$
\mathcal{L}=\frac{i}{2} \bar{\psi}^{H} \gamma^{\mu} \partial_{\mu} \psi^{H}-\frac{i}{2} \partial_{\mu} \bar{\psi}^{H} \gamma^{\mu} \psi^{H}-s J_{\mu} J^{\mu},
$$

and thus, non-linear Heisenberg equation reads $s^{2,29}$

$$
i \gamma^{\mu} \partial_{\mu} \psi^{H}-2 s\left(A+i B \gamma^{5}\right) \psi^{H}=0
$$

where $s$ stands for a constant which has dimension of (length $)^{2}$ and the physical amounts $A$ and $B$ are given in terms of the usual bilinear covariants associated with the Heisenberg spinor, given by (1) and (2), respectively. The Heisenberg spinor can be represented by a line in a two-dimensional plane $(\pi)$, where each axis is represented by the left-hand and right-hand spinors. ${ }^{2}$ In such a way, the Heisenberg spinor can be portrayed as the following identity:

$$
\psi^{H}=\psi_{L}^{H}+\psi_{R}^{H}
$$

In other words,

$$
\psi^{H}=\frac{1}{2}\left(\mathbb{1}+\gamma^{5}\right) \psi^{H}+\frac{1}{2}\left(\mathbb{1}-\gamma^{5}\right) \psi^{H}
$$

A particular class of solutions of the Heisenberg equation (11) is given by

$$
\partial_{\mu} \psi=\left(a J_{\mu}+b K_{\mu} \gamma^{5}\right) \psi
$$

with $a, b \in \mathbb{C}$ of dimensionality (length) $)^{2}, J_{\mu}$ and $K_{\mu}$ are covariant and irrotational currents. A $\psi$ that satisfies condition (14) also satisfies the Heisenberg equation of motion if $a$ and $b$ are such that $2 s=i(a-b)^{2}$ and shall be called RIM (restricted Inomata-McKinley) spinor. As was recently shown in Ref. 3, every Dirac spinor written in terms of RIM spinors belongs to class 1 within the Lounesto classification. In order that (14) be integrable, the constants $a$ and $b$ must obey the constraint $\operatorname{Re}(a)=\operatorname{Re}(b)$.

Hence, we are able to define $J^{2}=J_{\mu} J^{\mu}$ and consequently

$$
J_{\mu}=\partial_{\mu} S
$$

where

$$
S=\frac{1}{(a+\bar{a})} \ln \sqrt{J^{2}}
$$

represents a scalar, and similarly, we can write 
with

$$
K_{\mu}=\partial_{\mu} R,
$$

$$
R=\frac{1}{(b-\bar{b})} \ln \left(\frac{A-i B}{\sqrt{J^{2}}}\right),
$$

also being a scalar. (In order to make the notation compact, we define $\sqrt{J^{2}} \equiv J$.) From (14), we obtain for the left-hand and right-hand Heisenberg spinors

$$
\begin{aligned}
& \partial_{\mu} \psi_{L}^{H}=\left(a J_{\mu}+b K_{\mu}\right) \psi_{L}^{H}, \\
& \partial_{\mu} \psi_{R}^{H}=\left(a J_{\mu}-b K_{\mu}\right) \psi_{R}^{H} .
\end{aligned}
$$

Thus, to complete the program to be accomplished in the scope of this work, one is able to write an arbitrary spinor field, $\psi$, in terms of a $\pi$-plane decomposed Heisenberg spinor

$$
\psi=e^{F} \psi_{L}^{H}+e^{G} \psi_{R}^{H},
$$

and then, looking toward to write a linear theory in terms of a non-linear theory, one analyzes the properties encoded on the functions $F$ and $G$ in order that spinor (21) satisfies the Dirac equation. This is the prescription used in Ref. 2 to write Dirac spinors in terms of RIM-spinors. We will also follow this idea in Sec. III in order to write MDO spinors in terms of RIM-spinors.

\section{MASS-DIMENSION-ONE FERMIONS AND RIM-SPINORS}

Analogously as developed in Ref. 2, we analyze the possibility to write a MDO fermionic field ${ }^{5}$ in terms of the non-linear Heisenberg spinors. All the discussion is based on two fundamental equations, the non-linear Heisenberg equation and the "Dirac-like" equation for MDO fermions, ${ }^{4}$ which reads

$$
\left(i \gamma^{\mu} \partial_{\mu} \Xi \pm m \mathbb{1}\right) \lambda_{h}^{S / A}(\boldsymbol{x})=0,
$$

where the subscript $h$ stands for the helicity $h=\{ \pm, \mp\}$, the upper index $S / A$ stands for the self-conjugated and anti-self-conjugated spinors, respectively, under action of the charge conjugation operator $\left(\mathcal{C} \lambda_{h}^{S}=+\lambda_{h}^{S}\right.$ and $\left.\mathcal{C} \lambda_{h}^{A}=-\lambda_{h}^{A}\right)$, and the operator $\Xi$ in its matricial form is given by $^{9}$

$$
\Xi=\left(\begin{array}{cccc}
\frac{i p \sin \theta}{m} & \frac{-i(E+p \cos \theta) \mathrm{e}^{-i \phi}}{m} & 0 & 0 \\
\frac{i(E-p \cos \theta) \mathrm{e}^{i \phi}}{m} & \frac{-i p \sin \theta}{m} & 0 & 0 \\
0 & 0 & \frac{-i p \sin \theta}{m} & \frac{-i(E-p \cos \theta) \mathrm{e}^{-i \phi}}{m} \\
0 & 0 & \frac{i(E+p \cos \theta) \mathrm{e}^{i \phi}}{m} & \frac{i p \sin \theta}{m}
\end{array}\right) \text {, }
$$

where $p=|p|$. Then, we obtain the identity

$$
\lambda_{h}^{S / A}=\frac{1}{2}\left(\mathbb{1}+\gamma^{5}\right) \lambda_{h}^{S / A}+\frac{1}{2}\left(\mathbb{1}-\gamma^{5}\right) \lambda_{h}^{S / A}
$$

Expliciting the left- and right-handed components,

$$
\begin{aligned}
& \lambda_{R_{h}}^{S / A}=\frac{1}{2}\left(\mathbb{1}-\gamma_{5}\right) \lambda_{h}^{S / A}, \\
& \lambda_{L_{h}}^{S / A}=\frac{1}{2}\left(\mathbb{1}+\gamma_{5}\right) \lambda_{h}^{S / A} .
\end{aligned}
$$

We are now able to initiate the process to reach the decomposition (or representation) of the MDO spinors in terms of RIM-spinors, following the ideas of Subsection II B. First, one can write

$$
\lambda_{h}^{S / A}=e^{\vec{F}} \psi_{L_{h^{\prime}}}^{H}+e^{\vec{G}} \psi_{R_{h^{\prime}}}^{H},
$$

and, consequently, for the left- and right-handed components, we obtain

$$
\begin{aligned}
& \lambda_{L_{h}}^{S / A}=e^{\vec{F}} \psi_{L_{h^{\prime}}}^{H}, \\
& \lambda_{R_{h}}^{S / A}=e^{\vec{G}} \psi_{R_{h^{\prime}}}^{H} .
\end{aligned}
$$


The symbol " $\neg$ ” over $F$ and $G$, although commonly used to represent the dual of $\lambda$, is here simply to denote the functions related to $\lambda$ in the attempt to RIM-decompose such a spinor and does not have any relation to the dual of the field.

Following the program, the next step is to find the explicit form of $\vec{F}$ and $\vec{G}$ in order that $\lambda_{h}^{S / A}$ satisfies (22). By the same akin reasoning presented in Ref. 2 but now for the MDO spinors, we note that

$$
\partial_{\mu}=\partial_{\mu} S \frac{\partial}{\partial S}+\partial_{\mu} R \frac{\partial}{\partial R}
$$

Taking into account the relations in Eqs. (16) and (18), we are able to write (30) in this fashion

$$
\partial_{\mu}=J_{\mu} \frac{\partial}{\partial S}+K_{\mu} \frac{\partial}{\partial R}
$$

and therefore, one obtains

$$
\partial_{\mu} \lambda_{L_{h}}^{S / A}=\left(\frac{\partial \vec{F}}{\partial S} J_{\mu}+\frac{\partial \vec{F}}{\partial R} K_{\mu}\right) \lambda_{L_{h}}^{S / A}+\left(a J_{\mu}+b K_{\mu}\right) \lambda_{L_{h}}^{S / A}
$$

and

$$
\partial_{\mu} \lambda_{R_{h}}^{S / A}=\left(\frac{\partial \vec{G}}{\partial S} J_{\mu}+\frac{\partial \vec{G}}{\partial R} K_{\mu}\right) \lambda_{R_{h}}^{S / A}+\left(a J_{\mu}-b K_{\mu}\right) \lambda_{R_{h}}^{S / A} .
$$

Taking advantage of the Dirac-like equation, we multiply Eqs. (32) and (33) by $i \gamma^{\mu}$, and then using the fact that $\Xi^{2}=\mathbb{1}$ and $\left[\Xi, \gamma^{\mu} p_{\mu}\right]=0$, we have $^{30}$

$$
\begin{aligned}
i \gamma^{\mu} \partial_{\mu} \lambda_{h}^{S} & =i(A-i B)\left(\frac{\partial \vec{F}}{\partial S}-\frac{\partial \vec{F}}{\partial R}+(a-b)\right) \lambda_{R_{h}}^{S}+i(A+i B)\left(\frac{\partial \vec{G}}{\partial S}-\frac{\partial \vec{G}}{\partial R}+(a-b)\right) \lambda_{L_{h}}^{S} \\
& =m \Xi \lambda_{h}^{S} .
\end{aligned}
$$

Using the relations (for more information, refer to Appendix A) (A5)-(A8), one obtains the following set of equations:

$$
\begin{aligned}
& {\left[(A-i B)\left(\frac{\partial \vec{F}}{\partial S}-\frac{\partial \vec{F}}{\partial R}+(a-b)\right) \mathbb{1}+i m \Xi_{1}\right] \lambda_{R_{h}}^{S}=0,} \\
& {\left[(A+i B)\left(\frac{\partial \vec{G}}{\partial S}+\frac{\partial \vec{G}}{\partial R}+(a-b)\right) \mathbb{1}+i m \Xi_{2}\right] \lambda_{L_{h}}^{S}=0 .}
\end{aligned}
$$

At this stage, we freely summarized the notation and rewrite (23) as follows:

$$
\Xi=\left(\begin{array}{lr}
\Xi_{1} & 0_{2 \times 2} \\
0_{2 \times 2} & \Xi_{2}
\end{array}\right) .
$$

After a bit of straightforward calculation, the solutions for $\vec{F}(S, R)$ and $\vec{G}(S, R)$ functions are given by

$$
\begin{aligned}
& \vec{F}_{ \pm}(S, R) \equiv-2 i s R \pm \frac{p \sin \theta(A+i B) e^{-2(a+\bar{a}) S}}{2(a+\bar{a})}, \\
& \vec{G}_{ \pm}(S, R) \equiv+2 i s R \pm \frac{p \sin \theta(A-i B) e^{-2(a+\bar{a}) S}}{2(a+\bar{a})} .
\end{aligned}
$$

Note that

$$
A+i B=\frac{J^{2}}{A-i B}
$$

and from (16), we have

$$
J^{2}=e^{2(a+\bar{a}) S} .
$$


Therefore,

$$
\begin{aligned}
e^{\vec{F}_{-}} & =\exp \left[-2 i s R-\frac{1}{2} \frac{p \sin \theta}{(a+\bar{a})(A-i B)}\right], \\
e^{\vec{G}_{-}} & =\exp \left[+2 i s R-\frac{1}{2} \frac{p \sin \theta}{(a+\bar{a})(A+i B)}\right],
\end{aligned}
$$

then, with $\vartheta \equiv e^{2 i s R}$, we have

$$
\begin{aligned}
e^{\vec{F}_{-}} & =\frac{1}{\vartheta} \exp \left[-\frac{1}{2} \frac{p \sin \theta}{(a+\bar{a})(A-i B)}\right], \\
e^{\bar{G}_{-}} & =\vartheta \exp \left[-\frac{1}{2} \frac{p \sin \theta}{(a+\bar{a})(A+i B)}\right] .
\end{aligned}
$$

Following an analog prescription, we can write

$$
\begin{aligned}
e^{\bar{F}_{ \pm}} & =\frac{1}{\vartheta} \exp \left[ \pm \frac{1}{2} \frac{p \sin \theta}{(a+\bar{a})(A-i B)}\right], \\
e^{\bar{G}_{ \pm}} & =\vartheta \exp \left[ \pm \frac{1}{2} \frac{p \sin \theta}{(a+\bar{a})(A+i B)}\right] .
\end{aligned}
$$

In this manner, we finally write the MDO spinors in terms of RIM-spinors

$$
\lambda_{h}=\frac{1}{\vartheta} \exp \left[ \pm \frac{p \sin \theta}{2(a+\bar{a})(A-i B)}\right] \psi_{L_{h}}^{H}+\vartheta \exp \left[ \pm \frac{p \sin \theta}{2(a+\bar{a})(A+i B)}\right] \psi_{R_{h}}^{H}
$$

or, one is able to write the last expression in the fashion (replacing $p$ to $m$ )

$$
\lambda=\left(\sqrt{\frac{J}{A-i B}}\right)^{\rho} \exp \left[ \pm \frac{m \sin \theta}{4 \operatorname{Re}(a)(A-i B)}\right] \psi_{L}^{H}+\left(\sqrt{\frac{A-i B}{J}}\right)^{\rho} \exp \left[ \pm \frac{m \sin \theta}{4 \operatorname{Re}(a)(A+i B)}\right] \psi_{R}^{H}
$$

where we have defined $\rho \equiv \frac{\operatorname{Im}(a)-\operatorname{Im}(b)}{\operatorname{Im}(b)}=\frac{-2 s}{\operatorname{Im}(b)}$. Note that we omitted the upper index $S / A$ due to the fact that such spinors differ from a global phase. As a net result, we reach that the MDO fields can be freely represented as a combination of RIM-spinors which satisfy the non-linear Heisenberg equation.

\section{TWO-DIMENSIONAL SPINOR-SPACES: THE SPINOR-PLANE}

We start this section giving the definition of the spaces in which we will work on.

Definition 1. We denote by $\Pi^{H}$ the two-dimensional space whose set $\mathcal{B}=\left\{\Psi_{L}^{H}, \Psi_{R}^{H}\right\}$ (namely, the left- and right-handed components of the RIM-spinor $\Psi^{H}$ ) forms a basis. Analogously, we denote the spaces $\Pi^{D}$ (with basis $\mathcal{D}=\left\{\Psi_{L}^{D}, \Psi_{R}^{D}\right\}$ being formed by the components of the Dirac-RIM spinor) and $\Pi^{M}$ (with basis formed by the MDO-RIM components $\mathcal{M}=\left\{\lambda_{L}, \lambda_{R}\right\}$ ). These spaces will be called spinor-planes. $\mathcal{B}$, as

In order to achieve better organization, let us record that we can write Dirac spinors ${ }^{2} \Psi^{D}$ and MDO spinors $\lambda$ in the $\Pi^{H}$ space, via basis

$$
\begin{aligned}
\Psi^{D} & =\exp \left[\frac{i M}{(a+\bar{a}) J}\right] J^{2 \sigma}\left(\sqrt{\frac{J}{A-i B}} \Psi_{L}^{H}+\sqrt{\frac{A-i B}{J}} \Psi_{R}^{H}\right), \\
\lambda & =\exp \left[\frac{ \pm m \sin \theta}{4 \operatorname{Re}(a)(A-i B)}\right]\left(\sqrt{\frac{J}{A-i B}}\right)^{\rho} \Psi_{L}^{H}+\exp \left[\frac{ \pm m \sin \theta}{4 \operatorname{Re}(a)(A+i B)}\right]\left(\sqrt{\frac{A-i B}{J}}\right)^{\rho} \Psi_{R}^{H},
\end{aligned}
$$


with $J^{2 \sigma}=\exp \left\{\left[2 i s-\frac{1}{2}(b-\bar{b})\right] S\right\}=\exp \left[-i \frac{\operatorname{Im}(a)}{2 \operatorname{Re}(a)} \ln J\right]$. Now we will set the following notations for these complex numbers, for the sake of clarity:

$$
\begin{aligned}
& \alpha \equiv \exp \left[\frac{i M}{(a+\bar{a}) J}\right], \\
& \beta \equiv J^{2 \sigma}, \\
& \delta \equiv \sqrt{\frac{J}{A-i B}}, \\
& \epsilon \equiv\left(\sqrt{\frac{J}{A-i B}}\right)^{\rho}, \\
& \omega \equiv \exp \left[\frac{ \pm m \sin \theta}{4 \operatorname{Re}(a)(A-i B)}\right], \\
& \zeta \equiv \exp \left[\frac{ \pm m \sin \theta}{4 \operatorname{Re}(a)(A+i B)}\right] .
\end{aligned}
$$

In this fashion, one can denote the left- and right-handed components of the fields as

$$
\begin{aligned}
& \Psi_{L}^{D}=\alpha \beta \delta \Psi_{L}^{H}, \\
& \Psi_{R}^{D}=\alpha \beta \delta^{-1} \Psi_{R}^{H}, \\
& \lambda_{L}=\epsilon \omega \Psi_{L}^{H}, \\
& \lambda_{R}=\epsilon^{-1} \zeta \Psi_{R}^{H},
\end{aligned}
$$

which leads to

$$
\begin{aligned}
\lambda_{L} & =\chi_{1} \Psi_{L}^{D}, \\
\lambda_{R} & =\chi_{2} \Psi_{R}^{D}, \\
\Psi_{L}^{D} & =\chi_{1}^{-1} \lambda_{L}, \\
\Psi_{R}^{D} & =\chi_{2}^{-1} \lambda_{R},
\end{aligned}
$$

with the coefficients defined as $\chi_{1} \equiv \epsilon \omega \delta^{-1} \beta^{-1} \alpha^{-1}$ and $\chi_{2} \equiv \epsilon^{-1} \zeta \delta \beta^{-1} \alpha^{-1}$ being obviously invertible. These coefficients and their inverses are the tools that map Dirac-RIM spinors into MDO-RIM spinors and vice versa. After some straightforward calculations, one achieves an explicit form of those complex coefficients as

$$
\begin{gathered}
\chi_{1}=\left(\sqrt{\frac{J}{A-i B}}\right)^{\rho-1} \exp \left\{\frac{1}{2 \operatorname{Re}(a)}\left[ \pm \frac{m \sin \theta}{2(A-i B)}-i\left(\operatorname{Im}(a) \ln J+\frac{M}{J}\right)\right]\right\}, \\
\chi_{1}^{-1}=\left(\sqrt{\frac{A-i B}{J}}\right)^{\rho-1} \exp \left\{\frac{1}{2 \operatorname{Re}(a)}\left[\mp \frac{m \sin \theta}{2(A-i B)}+i\left(\operatorname{Im}(a) \ln J+\frac{M}{J}\right)\right]\right\}, \\
\chi_{2}=\left(\sqrt{\frac{A-i B}{J}}\right)^{\rho-1} \exp \left\{\frac{1}{2 \operatorname{Re}(a)}\left[ \pm \frac{m \sin \theta}{2(A+i B)}-i\left(\operatorname{Im}(a) \ln J+\frac{M}{J}\right)\right]\right\}, \\
\chi_{2}^{-1}=\left(\sqrt{\frac{J}{A-i B}}\right)^{\rho-1} \exp \left\{\frac{1}{2 \operatorname{Re}(a)}\left[\mp \frac{m \sin \theta}{2(A+i B)}+i\left(\operatorname{Im}(a) \ln J+\frac{M}{J}\right)\right]\right\} .
\end{gathered}
$$

This way, one can obtain

$$
\begin{aligned}
\lambda & =\frac{1}{2}\left[\chi_{1}\left(\mathbb{1}+\gamma^{5}\right)+\chi_{2}\left(\mathbb{1}-\gamma^{5}\right)\right] \Psi^{D}, \\
\Psi^{D} & =\frac{1}{2}\left[\chi_{1}^{-1}\left(\mathbb{1}+\gamma^{5}\right)+\chi_{2}^{-1}\left(\mathbb{1}-\gamma^{5}\right)\right] \lambda .
\end{aligned}
$$


If we define the matrices $M \equiv \frac{1}{2}\left[\chi_{1}\left(\mathbb{1}+\gamma^{5}\right)+\chi_{2}\left(\mathbb{1}-\gamma^{5}\right)\right]$ and $N \equiv \frac{1}{2}\left[\chi_{1}^{-1}\left(\mathbb{1}+\gamma^{5}\right)+\chi_{2}^{-1}\left(\mathbb{1}-\gamma^{5}\right)\right]$, this helps us to easily verify that $M N=N M=\mathbb{1}$, i.e., $N=M^{-1}$. Then, we have just proved the following:

Lemma 1. Let $\varphi_{D} \in \Pi^{D}$ and $\varphi_{\lambda} \in \Pi^{M}$. There exists a linear isomorphism $M: \Pi^{D} \rightarrow \Pi^{M}$, given by means of a matricial operator $M=\frac{1}{2}\left[\chi_{1}\left(\mathbb{I}+\gamma^{5}\right)+\chi_{2}\left(\mathbb{1}-\gamma^{5}\right)\right]$ such that

$$
\begin{aligned}
\varphi_{\lambda} & =M \varphi_{D}, \\
\varphi_{D} & =M^{-1} \varphi_{\lambda} .
\end{aligned}
$$

Lemma 1 shows a linear bijective (algebraic) map between special classes of MDO and Dirac fields, when both are decomposable in terms of RIM-spinors.

Note that an analog procedure can be done between all the other combinations of the spinor-spaces. Thus, using $(v, w)_{\mathcal{A}}$ as a notation for the coordinates of a given spinor in a basis $\mathcal{A}$ of a spinor-space $\Pi^{\mathcal{A}}$, for $\mathcal{A} \in\{\mathcal{B} \Leftrightarrow \mathcal{D} \Leftrightarrow \mathcal{M}\}$, one can represent $\Psi^{H}, \Psi^{D}$, and $\lambda$ as

$$
\begin{aligned}
\Psi^{H} & =(1,1)_{\mathcal{B}} & =\left(\alpha^{-1} \beta^{-1} \delta^{-1}, \alpha^{-1} \beta^{-1} \delta\right)_{\mathcal{D}} & =\left(\epsilon^{-1} \omega^{-1}, \epsilon \zeta^{-1}\right)_{\mathcal{M}}, \\
\Psi^{D} & =\left(\alpha \beta \delta, \alpha \beta \delta^{-1}\right)_{\mathcal{B}} & =(1,1)_{\mathcal{D}} & =\left(\chi_{1}^{-1}, \chi_{2}^{-1}\right)_{\mathcal{M}}, \\
\lambda & =\left(\epsilon \omega, \epsilon^{-1} \zeta\right)_{\mathcal{B}} & =\left(\chi_{1}, \chi_{2}\right)_{\mathcal{D}} & =
\end{aligned}
$$

Precisely, the construction of the (invertible) operators $L: \Pi^{H} \rightarrow \Pi^{D}$ and $Q: \Pi^{H} \rightarrow \Pi^{M}$ leads to matricial representations given by

$$
\begin{aligned}
& L=\frac{1}{2}\left[(\alpha \beta \delta)\left(\mathbb{1}+\gamma^{5}\right)+\left(\alpha \beta \delta^{-1}\right)\left(\mathbb{1}-\gamma^{5}\right)\right], \\
& Q=\frac{1}{2}\left[(\epsilon \omega)\left(\mathbb{1}+\gamma^{5}\right)+\left(\omega^{-1} \zeta\right)\left(\mathbb{1}-\gamma^{5}\right)\right]
\end{aligned}
$$

such that

$$
\begin{aligned}
\Psi^{D} & =L \Psi^{H}, \\
\Psi^{H} & =L^{-1} \Psi^{D}, \\
\lambda & =Q \Psi^{H}, \\
\Psi^{H} & =Q^{-1} \lambda .
\end{aligned}
$$

Then, we can state the following:

Lemma 2. Suppose the existence of a spinor-plane $\Pi^{S}$ with basis formed by left-and right-handed components of a given spinor $\psi=\psi_{L}+\psi_{R}$. If $\psi$ can be decomposed in terms of at least one of $\Psi^{H}, \Psi^{D}$, or $\lambda$ components with both coefficients non-vanishing (in other words, the decomposition is invertible), then it can be written in terms of any of those spinors, i.e., $\Pi^{S} \cong \Pi^{H} \cong \Pi^{D} \cong \Pi^{M}$.

Proof. It is trivial using the results of Lemma 1 and Eqs. (79)-(82).

Note that Lemma 1 is a corollary of Lemma 2.

Another fact that is worthwhile to mention is that $M, Q$, and $L$ as shown in Lemma 1 and Eqs. (77) and (78) are all diagonal (as, obviously, their inverses). This is because of the nature of the chirality projector operators, and we can define as follows:

Definition 2. We define $\mathfrak{M}$ as being the space of all matricial operators $R$ such that $\psi=R \varphi$, with $\psi, \varphi$ being spinors that may be decomposed in terms of RIM-spinors. The space $\mathfrak{M}$ has the set of projector operators $\left\{\frac{1}{2}\left(\mathbb{1}+\gamma^{5}\right), \frac{1}{2}\left(\mathbb{1}-\gamma^{5}\right)\right\}$ as the basis, working with complex coefficients to form elements of $\mathfrak{M}$, i.e.,

$$
\forall R \in \mathfrak{M}, \exists c_{1}, c_{2} \in \mathbb{C}: R=c_{1} \frac{1}{2}\left(\mathbb{1}+\gamma^{5}\right)+c_{2} \frac{1}{2}\left(\mathbb{1}-\gamma^{5}\right)
$$

Explicitly, $R=\operatorname{diag}\left(c_{2}, c_{2}, c_{1}, c_{1}\right)$.

It should be clear that, when $c_{1}, c_{2} \neq 0$, every $R \in \mathfrak{M}$ is invertible, with $\operatorname{diag}\left(c_{2}^{-1}, c_{2}^{-1}, c_{1}^{-1}, c_{1}^{-1}\right)=R^{-1} \in \mathfrak{M}$.

Finally, given the aspect of all those spinor-planes, we can understand them as being, in fact, exactly the same space, with the matrices $M, L, Q$ and their inverses being change-of-basis matrix operators between the basis $\mathcal{B}, \mathcal{D}$, and $\mathcal{M}$, with this being valid for every matrix $R \in \mathfrak{M}$ with other basis of the spinor-plane. This way, we can understand the space $\mathfrak{M}$ as being the space of all change-of-basis matrix operators in the spinor-plane. Then, we have found a two-dimensional space of all spinors that may be decomposed in terms of 
RIM-spinors (given its left- and right-handed components to form a basis on this space), equipped with a space of change-of-basis matrix operators.

\section{THE $s$-SPACE, THE SPINOR-PLANE, AND HOMOTOPIC FUNCTIONS}

Reference 2 analyzed carefully the domain of parameters $a$ and $b$, in order to avoid singularities on the potentials $S$ and $R$. Writing the complex numbers $a=a_{0} e^{i \phi_{1}}$ and $b=b_{0} e^{i \phi_{2}}$ in their polar forms, one is able to separate all the possible values for these complex numbers into (only) six disjoint domains as follows:

$$
\begin{aligned}
& \Omega_{1} \equiv W_{1} \otimes Z_{1}, \\
& \Omega_{2} \equiv W_{4} \otimes Z_{1}, \\
& \Omega_{3} \equiv W_{4} \otimes Z_{4}, \\
& \Omega_{4} \equiv W_{2} \otimes Z_{2}, \\
& \Omega_{5} \equiv W_{3} \otimes Z_{2}, \\
& \Omega_{6} \equiv W_{3} \otimes Z_{3},
\end{aligned}
$$

in which the intervals are defined as $W_{1}=\left(0, \frac{\pi}{2}\right), W_{2}=\left(\frac{\pi}{2}, \pi\right), W_{3}=\left(\pi, \frac{3 \pi}{2}\right), W_{4}=\left(\frac{3 \pi}{2}, 2 \pi\right)$ for $\phi_{1}$, with an analog definition for $Z_{1}, Z_{2}$, $Z_{3}$, and $Z_{4}$ as intervals of $\phi_{2}$. The point here is that for different choices of $a$ and $b$ in those six domains, one can construct different spinor configurations. Then, in order to make our explanations more clear, we define the s-space as follows:

Definition 3. Let $\Omega \equiv \bigcup_{i=1}^{6} \Omega_{i}$ be the space of all the feasible choices of parameters $\left(\phi_{1}, \phi_{2}\right)$ for $a=a\left(\phi_{1}\right)$ and $b=b\left(\phi_{2}\right)$ that define the Heisenberg constant $s=\frac{i(a-b)}{2}$ for the RIM solution (14) of the Heisenberg equation (11). We will call $\Omega$ the s-space.

Now we are able to introduce another interpretation for the two-dimensional spinor space, as we are dealing with in this work: fix a basis on this space, say $\mathcal{B}$, so we are in the "RIM-copy" of the spinor-plane. In this copy, the spinor $\Psi^{H}$ is a linear function: accurately, it is the identity function $y_{H}(x)=x$. Yet in this copy of the plane, we have $\Psi^{D}$ given by the function $y_{D}(x)=\left(\frac{A-i B}{J}\right) x$, and $\lambda$ given by $y_{\lambda}(x)=\left(\frac{A-i B}{J}\right)^{-2 \rho} \omega^{-1} \zeta x$. For both Dirac and MDO cases, we have the variable $x$ being defined via the $s$-space $\Omega$, i.e., $x=x\left(a\left(\phi_{1}\right), b\left(\phi_{2}\right)\right)$, and also $y=y\left(a\left(\phi_{1}\right), b\left(\phi_{2}\right)\right)$. But once a pair $\left(\phi_{1}, \phi_{2}\right) \in \Omega$ is fixed, all coordinates on the spinor-plane for every spinor are a pair $(x, y(x))$ in every basis.

In other words, Dirac, MDO, and RIM spinors (depending on which basis we are working on the spinor-plane) are implicit functions of $a$ and $b$ (or, via s-space, of $\phi_{1}$ and $\phi_{2}$ ), i.e., behaving like functions of the type

$$
\begin{aligned}
\varphi_{\mathcal{B}}: \Omega & \longrightarrow \Pi^{H} \\
\left(\phi_{1}, \phi_{2}\right) & \mapsto\left(f_{1}, f_{2}\right)_{\mathcal{B}},
\end{aligned}
$$

with $f_{1}, f_{2}$ being complex functions of the pair $\left(\phi_{1}, \phi_{2}\right) \in \Omega$. In a similar way, we can define $\varphi_{\mathcal{D}}: \Omega \rightarrow \Pi^{D}$ and $\varphi_{\mathcal{M}}: \Omega \rightarrow \Pi^{M}$. Of course, it is also valid for every spinor in the spinor-plane (this is guaranteed by Lemma 2 ).

It should be clear that both $\Psi^{D}$ and $\lambda$ are linear functions (also the identity function) when represented in their "own copies" of the spinorplane (i.e., when they are written in terms of the basis $\mathcal{D}$ and $\mathcal{M}$, respectively). In fact, it is true for every possible spinor (i.e., $\Pi^{S} \ni \psi=(x, x)_{\mathcal{S}}$ in the spinor-plane) $\psi$ as described in Lemma 2. Following this idea, one can think on the basis change being a deformation of the points (which are functions) on the spinor-plane, leading us to the attempt of construction of a homotopy on this space. Before initiating this, we need first to note that each point on the spinor-plane (in any fixed basis) can be written as $(x, y(x))$, with $y: \mathbb{C} \rightarrow \mathbb{C}$. Note that every $y=y(x)$ is a function on topological spaces, once $x\left(\phi_{1}, \phi_{2}\right)$ is set.

For us to begin the construction of the homotopy $H$, let, for instance, the Dirac spinor $\Psi^{D}$ be represented as $(x, f(x))_{\mathcal{D}}=(x, g(x))_{\mathcal{B}}$. Then, we know that $f(x)=x$ and $g(x)=\left(\frac{A-i B}{J}\right) x$. Now we need to find a continuous map $H: \mathbb{C} \times[0,1] \rightarrow \mathbb{C}$ such that $H(x, 0)=f(x)$ and $H(x, 1)=g(x)$ for all $x$. Defining

$$
H(x, t)=(1-t) f(x)+\operatorname{tg}(x)=\left[1+t\left(\frac{A-i B}{J}-1\right)\right] x,
$$

we see that it satisfies the conditions, and a remarkable result comes out: for each fixed value $j \in(0,1)$, the function $H(x, j) \equiv H_{j}(x)$ induces a new representation for $\Psi^{D}$ as a pair $\left(x, H_{j}(x)\right)_{\mathcal{A}} \in \Pi^{A}$, which corresponds to an intermediate copy $\Pi^{A}$ of the spinor-plane or, equivalently, it gives to the spinor-plane a basis $\mathcal{A}=\left\{\Psi_{L}^{A}, \Psi_{R}^{A}\right\}$ corresponding to the definition of an intermediate spinor $\Psi^{A}$. Noting this fact, and remembering the result of Lemma 2, we can state the following:

Theorem 1. Let $f=f(x)$ and $g=g(x)$ be functions such that $(x, f(x))_{\mathcal{A}_{0}}$ and $(x, g(x))_{\mathcal{A}_{1}}$ represent the same spinor on the spinor-plane by different basis $\mathcal{A}_{0}$ and $\mathcal{A}_{1}$. Then, it is possible to construct a homotopy $H(x, t)$ between $f$ and $g$ that defines an infinite family of spinors $\Psi^{A_{j}}$ (that 
can be decomposed in terms of RIM-spinors), with each spinor being represented by the identity function if the basis $\mathcal{A}_{j}=\left\{\Psi_{L}^{A_{j}}, \Psi_{R}^{A_{j}}\right\}$ is used (or equivalently, each spinor is represented by $\left.(x, x)_{\mathcal{A}_{j}}\right)$ for each fixed $t=j \in[0,1]$.

It is clear that one could construct all the spinors in the spinor-plane by just simply choosing a pair of complex numbers (with these numbers depending on the values on the s-space) $\left(c_{1}, c_{2}\right)$ and writing down, for instance, $\psi=c_{1} \psi_{L}^{H}+c_{2} \Psi_{R}^{H}=\left(c_{1}, c_{2}\right)_{\mathcal{B}}$, but using the result of Theorem 1, one can deform continuously the functions-coordinates between two specific spinors, instead of just choosing, without any criteria, complex numbers as the coordinates. In other words, this method provides a family of spinors which is related to each other by functions belonging to the same homotopy class.

Moreover, we can state another result, now concerning homotopy and spinors on a fixed basis:

Theorem 2. There exists a homotopic equivalence relation between any two spinors $\psi$ and $\varphi$ that can be written in terms of RIM-spinors.

Proof. Looking at Eq. (84), what happens is that for every pair $\left(\phi_{1}, \phi_{2}\right) \in \Omega$, we fix a complex number $x=f_{1}$, and then, another complex number $f_{2}=f_{2}\left(f_{1}\right)=f_{2}(x)$ is determined to form the pair $\left(f_{1}, f_{2}\right)$ which represents a spinor in a certain basis of the spinor-plane. Therefore, with the s-space $\Omega$ acting as a support-space to construct the set of all allowed complex numbers $x$, we can understand each spinor $\psi$ itself as a map

$$
\begin{aligned}
\psi: \mathbb{C} & \longrightarrow \mathbb{C}^{2} \\
x & \mapsto(x, y(x)) .
\end{aligned}
$$

Then, we can construct a homotopy $G_{\mathcal{A}}: \mathbb{C} \times[0,1] \rightarrow \mathbb{C}^{2}$ between two spinors $\psi=\left(x, y_{\psi}\right)_{\mathcal{A}}$ and $\varphi=\left(x, y_{\varphi}\right)_{\mathcal{A}}$ in a fixed basis $\mathcal{A}$, given by

$$
G_{\mathcal{A}}(x, t)=\left(x,(1-t) y_{\psi}+t y_{\varphi}\right) .
$$

Clearly, in a fixed basis $\mathcal{A}$, we have $G_{\mathcal{A}}(x, 0)=\left(x, y_{\psi}\right)=\psi$ and $G_{\mathcal{A}}(x, 1)=\left(x, y_{\varphi}\right)=\varphi$. Thereupon, $G_{\mathcal{A}}$ makes explicit an equivalence relation between the spinors $\psi$ and $\varphi$ themselves.

Again, we could simply construct "by hand" spinors in the spinor-plane defining points with the help of s-space $\Omega$, but the comprehensive result of Theorem 2 allows us to obtain representations $(x, y(x))$ of spinors, in a given basis, that are intermediary deformations of two known (homotopic related) spinors; i.e., one can think of equivalence homotopy classes of spinors.

One can remember the well-known proposition which states that, if $A$ is a convex subset of $\mathbb{R}^{n}$ and $X$ is any topological space, then any two continuous maps $f, g: X \rightarrow A$ are homotopic. Thus, the two theorems presented here show a particular (yet remarkable) result, namely, that these $f$ and $g$, with convenient choice of spaces $X$ and $A$, can represent spinor fields.

We will discuss these two theorems more in detail in Sec. VII.

\section{ON THE DIRAC DUAL, BILINEAR COVARIANTS AND LOUNESTO CLASSIFICATION}

\section{A. RIM-spinors and bilinear covariants}

It is well known ${ }^{2,3}$ that RIM-spinors $\Psi^{H}$ are necessarily regular spinors; otherwise, it would be possible to have $A=0=B$ (with $A=\Psi^{H} \Psi^{H}$ and $B=i \Psi^{H} \gamma^{5} \Psi^{H}$ ), and then, the Heisenberg non-linear equation would reduce to the ordinary linear Dirac equation. Because of that, it seems that the possibility to have only one of the bilinears $A=0$ or $B=0$ (i.e., type- 2 and type- 3 RIM-spinors) is perfectly feasible since it remains intact the non-linear aspect of the Heisenberg equation. However,

Lemma 3. The RIM-spinors $\Psi^{H}$ are necessarily type-1 in Lounesto classification (i.e., $A, B \neq 0$ ).

Proof. First, note that $R=(b-\bar{b})^{-1} \ln \left(\frac{A-i B}{J}\right)$, which means, in particular, that $(b-\bar{b}) \neq 0$. In fact, by (17), otherwise, we would end up with $K_{\mu} \rightarrow \infty$, leading to an unphysical result. Now, in Ref. 2, it is claimed that $J_{\mu}$ and $K_{\mu}$ constitute a basis for vectors constructed by the derivative $\partial_{\mu}$ operating on functionals of $\Psi^{H}$, and one has the following equations (which are valid for every $\mu$ ):

$$
\begin{aligned}
& \partial_{\mu} A=(a+\bar{a}) A J_{\mu}+i(b-\bar{b}) B K_{\mu}, \\
& \partial_{\mu} B=(a+\bar{a}) B J_{\mu}+i(b-\bar{b}) A K_{\mu} .
\end{aligned}
$$

Suppose that $A=0$ and $B \neq 0$. Then, Eq. (88) gives $0=i(b-\bar{b}) B K_{\mu}$, which is a contradiction, since $\Psi^{H}$ is a regular spinor and we cannot have $K_{\mu}=0, \forall \mu$. Thus, $\Psi^{H}$ cannot be type-3. Moreover, if we suppose that $A \neq 0$ and $B=0$, then Eq. (89) provides $0=i(b-\bar{b}) A K_{\mu}$, an analog contradiction, and we conclude that $\Psi^{H}$ cannot be type-2 by the same reason as before. Therefore, we conclude that $A, B \neq 0$ and $\Psi^{H}$ is a regular type-1 spinor. 
We can extract more information about $A$ and $B$ from the explicit form of the scalar $R$. In fact, we can note that $(A-i B) \neq 0$ and $J \equiv \sqrt{J^{2}} \neq 0$. Then, remembering that $J^{2}=(A-i B)(A+i B)$, we also conclude that $(A+i B) \neq 0$. Now, let us represent a RIM-spinor as $\Psi^{H}=\left(\begin{array}{llll}\Psi_{11} & \Psi_{12} & \Psi_{21} & \Psi_{22}\end{array}\right)^{T}$. Let us define

$$
\begin{aligned}
& A_{1} \equiv \Psi_{21}^{*} \Psi_{11}+\Psi_{22}^{*} \Psi_{12}, \\
& A_{2} \equiv \Psi_{11}^{*} \Psi_{21}+\Psi_{12}^{*} \Psi_{22},
\end{aligned}
$$

with $r^{*}$ denoting the complex conjugate of $r \in \mathbb{C}$. Then, it is straightforward to see that

$$
\begin{aligned}
& A=A_{1}+A_{2}, \\
& B=i\left(-A_{1}+A_{2}\right) .
\end{aligned}
$$

With this in hand, since $A, B \neq 0$, we conclude that $A_{1} \neq \pm A_{2}$. Besides, $A+i B=2 A_{1} \neq 0 \Rightarrow A_{1} \neq 0$, and $A-i B=2 A_{2} \neq 0 \Rightarrow A_{2} \neq 0$.

These conditions will turn into strong constraints on RIM-decomposable bilinear covariants.

\section{B. RIM-decomposable spinors and bilinear covariants}

Let $\psi=\psi_{L}+\psi_{R}$ be a spinor that can be decomposed in terms of RIM-spinors, so there exists a matrix $R \in \mathfrak{M}$ such that $\psi=R \Psi^{H}$. In this case, we can write $R=\operatorname{diag}\left(r_{1}, r_{1}, r_{2}, r_{2}\right)$ with decomposition $\psi=r_{1} \Psi_{L}^{H}+r_{2} \Psi_{R}^{H}$. Suppose that its dual is constructed in the Dirac fashion $\bar{\psi}=\psi^{\dagger} \gamma^{0}$. Let us represent the bilinear covariants associated with $\psi$ as $A_{\psi}, B_{\psi}, \mathbf{J}_{\psi}, \mathbf{K}_{\psi}, \mathbf{S}_{\psi}$.

We want to categorize all RIM-decomposable spinors in the Lounesto classification. In order to do that, initially, we need to know the conditions for $\mathbf{J}_{\psi} \neq 0$ because it is an imposition in the aforementioned classification. Since $\psi=R \Psi^{H}$, we can write

$$
J_{\psi}^{\mu}=\left(\Psi^{H}\right)^{\dagger} R^{\dagger} \gamma^{0} \gamma^{\mu} R \Psi^{H}
$$

Representing $\Psi^{H}=\left(\Psi_{1} \Psi_{2}\right)^{T}$ with $\Psi_{j} \equiv\left(\Psi_{j 1} \Psi_{j 2}\right)^{T}$ for $j \in\{1,2\}$, we obtain

$$
\begin{aligned}
J_{\psi}^{0} & =\left|\Psi_{1}\right|^{2}\left|r_{1}\right|^{2}+\left|\Psi_{2}\right|^{2}\left|r_{2}\right|^{2}, \\
J_{\psi}^{1} & =-\left|r_{1}\right|^{2}\left(\Psi_{12}^{*} \Psi_{11}+\Psi_{11}^{*} \Psi_{12}\right)+\left|r_{2}\right|^{2}\left(\Psi_{22}^{*} \Psi_{21}+\Psi_{21}^{*} \Psi_{22}\right), \\
J_{\psi}^{2} & =i\left[-\left|r_{1}\right|^{2}\left(\Psi_{12}^{*} \Psi_{11}-\Psi_{11}^{*} \Psi_{12}\right)+\left|r_{2}\right|^{2}\left(\Psi_{22}^{*} \Psi_{21}-\Psi_{21}^{*} \Psi_{22}\right)\right], \\
J_{\psi}^{3} & =-\left|r_{1}\right|^{2}\left(\left|\Psi_{11}\right|^{2}-\left|\Psi_{12}\right|^{2}\right)+\left|r_{2}\right|^{2}\left(\left|\Psi_{21}\right|^{2}-\left|\Psi_{22}\right|^{2}\right) .
\end{aligned}
$$

One has to look for the conditions that lead to $J_{\psi}^{\mu}=0, \forall \mu \in\{0,1,2,3\}$, simultaneously. These conditions will form the exact conditions that we have to avoid. We have to verify the components $J_{\psi}^{\mu}$ one by one. Thus, as a start, in order to reach $J_{\psi}^{0}=0$, one finds three options:

(i) $\quad r_{1} \neq 0, r_{2}=0$ and $\left|\Psi_{1}\right|^{2}=0$ (which, by symmetry, is equivalent to $r_{2} \neq 0, r_{1}=0$ and $\left|\Psi_{2}\right|^{2}=0$ ).

(ii) $\left|\Psi_{1}\right|^{2}=0=\left|\Psi_{2}\right|^{2}$.

(iii) $r_{1}=0=r_{2}$.

Obviously, $r_{1}=0=r_{2}$ is not an allowed option, as it leads to $\psi=0$ with all bilinear covariants vanishing, which is not interesting. Note that option (ii) leads to $\Psi^{H}=0=\psi$; then, we descart it. Now, we have to analyze the case of option (i). In fact, one can easily verify that condition (i) simultaneously vanishes Eqs. (95)-(98), i.e.,

$$
\mathbf{J}_{\psi}=0 \Leftrightarrow(i)
$$

Then, we conclude that we have to avoid condition (i).

The scalar $A_{\psi}=\bar{\psi} \psi$ and the pseudo-scalar $B_{\psi}=i \bar{\psi} \gamma^{5} \psi$ can both be written in terms of the four components of $\Psi^{H}$ as

$$
\begin{aligned}
& A_{\psi}=\left(r_{1} r_{2}^{*}\right)\left(\Psi_{21}^{*} \Psi_{11}+\Psi_{22}^{*} \Psi_{12}\right)+\left(r_{1}^{*} r_{2}\right)\left(\Psi_{11}^{*} \Psi_{21}+\Psi_{12}^{*} \Psi_{22}\right), \\
& B_{\psi}=i\left[-\left(r_{1} r_{2}^{*}\right)\left(\Psi_{21}^{*} \Psi_{11}+\Psi_{22}^{*} \Psi_{12}\right)+\left(r_{1}^{*} r_{2}\right)\left(\Psi_{11}^{*} \Psi_{21}+\Psi_{12}^{*} \psi_{22}\right)\right] .
\end{aligned}
$$

For the particular case of $r_{1}, r_{2} \in \mathbb{R}$ (in other words, if $R$ is real), we have the interesting fact $A_{\psi}=\left(r_{1} r_{2}\right) A$ and $B_{\psi}=\left(r_{1} r_{2}\right) B$, i.e., $A_{\psi} \propto A$ and $B_{\psi} \propto B$, and we have that $\psi=r_{1} \Psi_{L}^{H}+r_{2} \Psi_{R}^{H}$ is always a type-1 spinor when $r_{1}, r_{2} \in \mathbb{R}-\{0\}$.

In order to have $\psi$ as a RIM-decomposable spinor, we have two options (remember that it is equivalent to $r_{2} \neq 0$ and $r_{1}=0$ ): $r_{1}, r_{2} \neq 0$ or $r_{1} \neq 0, r_{2}=0\left(r_{2} \neq 0, r_{1}=0\right)$. Now, note that the second option cannot happen with $\left|\Psi_{1}\right|^{2}=0\left(\left|\Psi_{2}\right|^{2}=0\right)$ occurring since it would lead to condition (i). Then, for the sake of clarity, we will split our study into two cases. In the case $r_{1} \neq 0, r_{2}=0$, we will show that 
Lemma 4. For a RIM-decomposable spinor $\psi$ such that $\bar{\psi}=\psi^{\dagger} \gamma^{0}$, we have

$$
\mathbf{J}_{\psi} \neq 0 \Rightarrow\left(\mathbf{K}_{\psi} \neq 0 \text { and } \mathbf{S}_{\psi}=0\right)
$$

every time the conditions $r_{1} \neq 0$ and $r_{2}=0$ (or, equivalently, $r_{2} \neq 0$ and $r_{1}=0$ ) are satisfied.

Proof. We will look for the conditions to make $\mathbf{K}_{\psi}=0$ and $\mathbf{S}_{\psi}=0$ in this case. First, let us analyze $\mathbf{K}_{\psi}$. Analogously to what was made to reach Eqs. (95)-(98), we obtain

$$
K_{\psi}^{0}=\left|\Psi_{1}\right|^{2}\left|r_{1}\right|^{2}-\left|\Psi_{2}\right|^{2}\left|r_{2}\right|^{2} .
$$

Now, suppose $r_{2}=0$ (then, $r_{1} \neq 0$ ). Thus, in order to have $K_{\psi}^{0}=\left|\Psi_{1}\right|^{2}\left|r_{1}\right|^{2}=0$, one must have $\left|\Psi_{1}\right|^{2}=0$. But it would lead to condition (i). Therefore, by relation (99), in this case, we cannot have $\mathbf{K}_{\psi}=0$.

Now, let us analyze $\mathbf{S}_{\psi}$. In the same fashion, we can write

$$
\begin{aligned}
& S_{\psi}^{01}=-i\left[\left(r_{2}^{*} r_{1}\right)\left(\Psi_{22}^{*} \Psi_{11}+\Psi_{21}^{*} \Psi_{12}\right)-\left(r_{1}^{*} r_{2}\right)\left(\Psi_{12}^{*} \Psi_{21}+\Psi_{11}^{*} \Psi_{22}\right)\right], \\
& S_{\psi}^{02}=\left(r_{2}^{*} r_{1}\right)\left(\Psi_{22}^{*} \Psi_{11}-\Psi_{21}^{*} \Psi_{12}\right)-\left(r_{1}^{*} r_{2}\right)\left(\Psi_{12}^{*} \Psi_{21}-\Psi_{11}^{*} \Psi_{22}\right), \\
& S_{\psi}^{03}=-i\left[\left(r_{2}^{*} r_{1}\right)\left(\Psi_{21}^{*} \Psi_{11}-\Psi_{22}^{*} \Psi_{12}\right)-\left(r_{1}^{*} r_{2}\right)\left(-\Psi_{11}^{*} \Psi_{21}+\Psi_{12}^{*} \Psi_{22}\right)\right], \\
& S_{\psi}^{12}=\left(r_{2}^{*} r_{1}\right)\left(\Psi_{21}^{*} \Psi_{11}-\Psi_{22}^{*} \Psi_{12}\right)+\left(r_{1}^{*} r_{2}\right)\left(\Psi_{11}^{*} \Psi_{21}-\Psi_{12}^{*} \Psi_{22}\right), \\
& S_{\psi}^{13}=-i\left[\left(r_{2}^{*} r_{1}\right)\left(\Psi_{22}^{*} \Psi_{11}-\Psi_{21}^{*} \Psi_{12}\right)+\left(r_{1}^{*} r_{2}\right)\left(\Psi_{12}^{*} \Psi_{21}-\Psi_{11}^{*} \Psi_{22}\right)\right], \\
& S_{\psi}^{23}=\left(r_{2}^{*} r_{1}\right)\left(\Psi_{22}^{*} \Psi_{11}+\Psi_{21}^{*} \Psi_{12}\right)+\left(r_{1}^{*} r_{2}\right)\left(\Psi_{12}^{*} \Psi_{21}+\Psi_{11}^{*} \Psi_{22}\right) .
\end{aligned}
$$

Again, without loss of generalization, suppose $r_{2}=0$ (and, so, $r_{1} \neq 0$ ). In this case, it is obvious that we always have $S_{\psi}^{\mu v}=0$, i.e., $\mathbf{S}_{\psi}=0$, and it does not depend on any condition for the components $\Psi_{i j}$ whatsoever.

To summarize, we have found that $\mathbf{J}_{\psi} \neq 0 \Rightarrow \mathbf{K}_{\psi} \neq 0$ and $\mathbf{J}_{\psi} \neq 0 \Rightarrow \mathbf{S}_{\psi}=0$, when $r_{1} \neq 0$ and $r_{2}=0$, which can be written as $\mathbf{J}_{\psi} \neq 0 \Rightarrow\left(\mathbf{K}_{\psi} \neq 0\right.$ and $\left.\mathbf{S}_{\psi}=0\right)$. This ends the proof.

Now, note that, if $r_{1} \neq 0$ and $r_{2}=0$, then $A_{\psi}=0=B_{\psi}$ : in other words, in this case, we are dealing necessarily with singular spinors. But, using Lemma 4, we classify these spinors on the spinor-plane by the Lounesto classification, and if $r_{1} \neq 0$ and $r_{2}=0$, then we are dealing with type-6 singular spinors (see Subsection II A).

What about other situations that could lead to singular RIM-decomposable spinors? Let us see. In fact, the other option left is to have $r_{1}$, $r_{2} \neq 0$. Note that, looking at definitions (90) and (91) and Eqs. (100) and (101), it is straightforward to see that we can write

$$
\begin{aligned}
& A_{\psi}=\left(r_{1} r_{2}^{*}\right) A_{1}+\left(r_{1}^{*} r_{2}\right) A_{2}, \\
& B_{\psi}=i\left[-\left(r_{1} r_{2}^{*}\right) A_{1}+\left(r_{1}^{*} r_{2}\right) A_{2}\right] .
\end{aligned}
$$

Then, as a last try, if we choose $r_{1}, r_{2} \neq 0$, we see that we cannot simultaneously have $A_{\psi}=0=B_{\psi}$; thus, $\psi$ is a regular spinor and $\mathbf{K}_{\psi}$, $\mathbf{S}_{\psi} \neq 0$. Indeed, if we impose $A_{\psi}=0=B_{\psi}$ with $r_{1}, r_{2} \neq 0$, then $\left(r_{1} r_{2}^{*}\right) A_{1}=-\left(r_{1}^{*} r_{2}\right) A_{2}$ and $\left(r_{1} r_{2}^{*}\right) A_{1}=+\left(r_{1}^{*} r_{2}\right) A_{2}$, which has no solution once we know that $A_{1}, A_{2} \neq 0$. Note that this implies that we cannot have type- 4 and type- 5 singular RIM-decomposable spinors at all, since this exhausts all possibilities for $r_{1}$ and $r_{2}$ in the construction of a non-null $\psi$. Moreover, we have shown here that a decomposition leading to a singular spinor needs to satisfy $r_{1} \neq 0$ and $r_{2}=0$, and having $r_{1} \neq 0$ and $r_{2}=0$ is sufficient in order to have a decomposition leading to a singular spinor. Therefore, we can state the following:

Proposition 1. Suppose $\psi=\mathrm{R} \Psi^{H}$, with $R=\operatorname{diag}\left(r_{1}, r_{1}, r_{2}, r_{2}\right) \in \mathfrak{M}$ such that $\psi=r_{1} \Psi_{L}^{H}+r_{2} \Psi_{R}^{H}$, satisfying $\boldsymbol{J}_{\psi} \neq 0$ and $\bar{\psi}=\psi^{\dagger} \gamma^{0}$. Then, the statements below are equivalent:

(i) $\psi$ is a singular spinor.

(ii) $\psi$ is a type-6 spinor.

(iii) $r_{1}=0$ or $r_{2}=0$ (but not both).

(iv) $\quad \psi$ is projected only in $\left(0, \frac{1}{2}\right)$ or $\left(\frac{1}{2}, 0\right)$ representation, i.e., $\psi \propto \Psi_{L}^{H}$ or $\psi \propto \Psi_{R}^{H}$.

We have realized, thus, that once we are setting $r_{1}, r_{2} \neq 0$, we are dealing with regular spinors. In this case, we have other three options to verify: type-1 $\left(A_{\psi}, B_{\psi} \neq 0\right)$, type-2 $\left(A_{\psi} \neq 0\right.$ and $\left.B_{\psi}=0\right)$, and type-3 $\left(A_{\psi}=0\right.$ and $\left.B_{\psi} \neq 0\right)$. Since we know that $\Psi^{H}$ itself and the Dirac RIMdecomposable field $\Psi^{D}$ are both type- $1,{ }^{3}$ we only have to check the other two possibilities. First, if we set $A_{\psi}=0$, then $\left(r_{1} r_{2}^{*}\right) A_{1}=-\left(r_{1}^{*} r_{2}\right) A_{2}$ $\neq 0$, and we can write $B_{\psi}=2 i\left(r_{1}^{*} r_{2}\right) A_{2}=-2 i\left(r_{1} r_{2}^{*}\right) A_{1} \neq 0$, then it is possible to have type-2 RIM-decomposable spinors. Analogously, if we set $B_{\psi}=0$, then $\left(r_{1} r_{2}^{*}\right) A_{1}=\left(r_{1}^{*} r_{2}\right) A_{2} \neq 0$, and it leads to $A_{\psi}=2 i\left(r_{1}^{*} r_{2}\right) A_{2}=2 i\left(r_{1} r_{2}^{*}\right) A_{1} \neq 0$, which means that it is also possible to have type-3 RIM-decomposable spinors. With this in hands, we state that

Proposition 2. Suppose $\psi=R \Psi^{H}=r_{1} \Psi_{L}^{H}+r_{2} \Psi_{R}^{H}$, with $R=\operatorname{diag}\left(r_{1}, r_{1}, r_{2}, r_{2}\right) \in \mathfrak{M}$, satisfying $\boldsymbol{J}_{\psi} \neq 0$ and $\bar{\psi}=\psi^{\dagger} \gamma^{0}$. Then, $\psi$ is a regular spinor if, and only if, $r_{1}, r_{2} \neq 0$. Yet, in this case, we have that 
(i) $\quad \psi$ is a type-1 spinor if, and only if, $A \neq-i B\left(\frac{r_{1} r_{2}^{*} \pm r_{1}^{*} r_{2}}{r_{1} r_{2}^{*} \mp r_{1}^{*} r_{2}}\right)$.

(ii) $\quad \psi$ is a type-2 spinor if, and only if, $\left(r_{1}^{*} r_{2}\right)^{2} \neq\left(r_{1} r_{2}^{*}\right)^{2}$ and $A=-i B\left(\frac{r_{1} r_{2}^{*}+r_{1}^{*} r_{2}}{r_{1} r_{2}^{*}-r_{1}^{*} r_{2}}\right)$.

(iii) $\psi$ is a type-3 spinor if, and only if, $\left(r_{1}^{*} r_{2}\right)^{2} \neq\left(r_{1} r_{2}^{*}\right)^{2}$ and $A=-i B\left(\frac{r_{1} r_{2}^{*}-r_{1}^{*} r_{2}}{r_{1} r_{2}^{*}+r_{1}^{*} r_{2}}\right)$.

Proof. First, we have already seen that, in the hypothesis of this proposition, the condition $r_{1}, r_{2} \neq 0$ is necessary and sufficient for $\psi$ to be a regular spinor: in fact, this particular result can be understood as a corollary of Proposition 1 . Now, noting that $A_{1}=\frac{A+i B}{2}$ and $A_{2}=\frac{A-i B}{2}$, one is able to write

$$
\begin{aligned}
A_{\psi} & =\frac{1}{2}\left[A\left(r_{1} r_{2}^{*}+r_{1}^{*} r_{2}\right)+i B\left(r_{1} r_{2}^{*}-r_{1}^{*} r_{2}\right)\right], \\
B_{\psi} & =-\frac{i}{2}\left[A\left(r_{1} r_{2}^{*}-r_{1}^{*} r_{2}\right)+i B\left(r_{1} r_{2}^{*}+r_{1}^{*} r_{2}\right)\right] .
\end{aligned}
$$

We know that $A_{1}, A_{2} \neq 0$. Yet, one cannot reach $\left(r_{1} r_{2}^{*}-r_{1}^{*} r_{2}\right)=0=\left(r_{1} r_{2}^{*}+r_{1}^{*} r_{2}\right)$ with $r_{1}, r_{2} \neq 0$ : in fact, it would lead to $A_{\psi}=0=B_{\psi}$, an unattainable case here, as we have seen.

Then, in order to reach $B_{\psi}=0$, we need to have $A\left(r_{1} r_{2}^{*}-r_{1}^{*} r_{2}\right)+i B\left(r_{1} r_{2}^{*}+r_{1}^{*} r_{2}\right)=0$. Moreover, one cannot have $\left(r_{1} r_{2}^{*}+r_{1}^{*} r_{2}\right)=0$ or $\left(r_{1} r_{2}^{*}-r_{1}^{*} r_{2}\right)=0$ isolated because it will never make $B_{\psi}=0$; in other words, $\left(r_{1}^{*} r_{2}\right)^{2} \neq\left(r_{1} r_{2}^{*}\right)^{2}$. Thus, the only option left is $A\left(r_{1} r_{2}^{*}-r_{1}^{*} r_{2}\right)=-i B\left(r_{1} r_{2}^{*}+r_{1}^{*} r_{2}\right)$, which implies that $A=-i B\left(\frac{r_{1} r_{2}^{*}+r_{1}^{*} r_{2}}{r_{1} r_{2}^{*}-r_{1}^{*} r_{2}}\right)$. In this case, it is guaranteed that $A_{\psi} \neq 0$. This proves item (ii).

Now, if one wants to have $A_{\psi}=0$, for analog reasons as the case above, we have $\left(r_{1}^{*} r_{2}\right)^{2} \neq\left(r_{1} r_{2}^{*}\right)^{2}$ and $A\left(r_{1} r_{2}^{*}-r_{1}^{*} r_{2}\right)=-i B\left(r_{1} r_{2}^{*}+r_{1}^{*} r_{2}\right)$. It leads to $A=-i B\left(\frac{r_{1} r_{2}^{*}+r_{1}^{*} r_{2}}{r_{1} r_{2}^{*}-r_{1}^{*} r_{2}}\right)$ (with $B_{\psi} \neq 0$ guaranteed), which proves item (iii).

So far, we have seen that the only way to vanish $A_{\psi}$ or $B_{\psi}$ without vanishing both at the same time is to have $A\left(r_{1} r_{2}^{*} \mp r_{1}^{*} r_{2}\right)$ $=-i B\left(r_{1} r_{2}^{*} \pm r_{1}^{*} r_{2}\right)$. Then, we conclude that we cannot have these conditions valid in order to keep both $A_{\psi}, B_{\psi} \neq 0$, i.e., having $A \neq-i B \frac{\left(r_{1} r_{2}^{*} \pm r_{1}^{*} r_{2}\right)}{\left(r_{1} r_{2}^{*} \mp r_{1}^{*} r_{2}\right)}$ is equivalent to say that $\psi$ can only be type-1, proving item (i).

Indeed, $\Psi^{H}$ has $r_{1}=r_{2}=1$, and Proposition 2 trivially confirms that $\Psi^{H}$ is type-1, with $A_{\Psi^{H}}=A$ and $B_{\Psi^{H}}=B$ being easily obtained by Eqs. (112) and (113), as expected. As another example, for the Dirac spinor $\Psi^{D}$, we have $r_{1}=\alpha \beta \delta$ and $r_{2}=\alpha \beta \delta^{-1}$ as defined in Sec. IV, and one can verify that $\frac{r_{1}+r_{2}}{r_{1}-r_{2}}=-i\left(\frac{A}{B}\right) \Rightarrow-i B\left(\frac{r_{1}+r_{2}}{r_{1}-r_{2}}\right)=-A \neq A$, and $\frac{r_{1}-r_{2}}{r_{1}+r_{2}}=i\left(\frac{B}{A}\right) \Rightarrow-i B\left(\frac{r_{1}-r_{2}}{r_{1}+r_{2}}\right)=\frac{B^{2}}{A} \neq A$, which confirms that $\Psi^{D}$ is also type- 1 .

Summarizing the results of this section, Propositions 1 and 2 provide an easy method to separate all spinors $\psi$ allowed in the spinorplane (with dual defined as $\left.\bar{\psi}=\psi^{\dagger} \gamma^{0}\right)$ in the Lounesto classification by just looking at their coefficients $\left(r_{1}, r_{2}\right)_{\mathcal{B}}$ in the "RIM-copy" $\Pi^{H}$ : if both coefficients are non-vanishing, then the spinor is regular (with an easy way to verify if it is type-1, type-2 or type-3: simply divide the sum of the coefficients by the difference-and the difference by the sum-and multiply by $-i B$ in order to verify if it is equal to $A$ ), while if one (and only one) of the coefficients is zero, then it is a singular type- 6 spinor, with no need to the often hard work of construction of all the bilinear covariants. As we cannot have $r_{1}=0=r_{2}$, all feasible cases are contemplated.

\section{FINAL REMARKS}

The second main result of Ref. 3, concerning exotic spinor fields, allows us to state that all spinors belonging to the spinor-plane that has a dynamic equation are not exotic spinors: i.e., the underlying topology of the space-time $M$ of which these spinors may emerge is trivial, in the sense that it has a trivial fundamental group $\pi_{1}(M)=0$. In particular, this spinor-plane accommodates a bijective linear map between special classes (i.e., both being RIM-decomposable and, therefore, non-exotic) of MDO and Dirac spinors. This mapping is quite natural, as it uses RIM-spinors as a fundamental element making the mediation between Dirac and MDO fields. Although this mapping has some constraints imposed in the fields themselves (they had to be RIM-decomposable), one does not have to work with the bilinear covariants, which is often a hard situation to deal with when we study MDO spinors since they do not necessarily fit in the usual Lounesto classification. Therefore, the mapping developed here transcends the problem of the Lounesto classification of MDO spinors.

Among the outcomes of this work, we emphasize that Theorem 1 is an exhaustive result: it gives not only the possibility to write down explicitly all possible spinors that can be decomposed by RIM-spinors, by giving the left- and right-handed components of each of them, but it also makes explicit an equivalence relation (via the homotopy $H$ ) between all the functions that represent spinor-plane coordinates. On the 
other hand, Theorem 2 is another robust result, providing a way to deform spinors in the spinor-plane, enabling the composition and the eventual classification of equivalence classes of homotopic spinors via the homotopies $G_{\mathcal{A}}$.

The two theorems are related, in the sense that both treat the subject of writing down explicit forms of representing the spinors that can be written in terms of RIM-spinors, by showing a homotopic equivalence relation. The main difference between them, which indeed complements each other, lies on the fact that Theorem 1 provides a method to obtain the left- and right-handed components of the spinors by constructing basis for the spinor-plane, while Theorem 2 supplies a way to obtain points (the spinors themselves) in a given fixed basis. In other words, in Theorem 1, we are continuously deforming the spinor-plane itself (obtaining new basis for the space), while in Theorem 2, we are continuously deforming the points of the plane in a fixed basis (obtaining intermediate spinors between two fixed ones).

The understanding of the very nature of spinors is a field of study under development, which is as significant in Physics as in Mathematics. Theorems 1 and 2 may be the beginning of a new way to look at the construction of spinors, opening the possibility to the discovery of interesting relations via homotopy theory, which is perhaps one of the most important ideas behind algebraic topology.

Propositions 1 and 2 facilitate the categorization of RIM-decomposable spinors $\psi$, which has $\bar{\psi}=\psi^{\dagger} \gamma^{0}$, in the Lounesto classification: they provide a complete and easy way to determine how these spinors are classified in the Lounesto classification when their dual is defined in the Dirac fashion. In fact, they connect the coefficients of their decomposition (or, in other words, their coordinates on the spinor-plane given in the basis $\mathcal{B}=\left\{\Psi_{L}^{H}, \Psi_{R}^{H}\right\}$ ) directly with the Lounesto classification, avoiding the construction of all bilinear covariants and the often laborious process of checking which of them are null and which ones are not. In particular, Proposition 2 is a generalization of Lemma 1 in Ref. 3, which states that every Dirac spinor decomposable in terms of RIM-spinors is type-1 in the Lounesto classification.

It is worthwhile to make clear that the core of all results of this work is in the RIM-decomposition itself, in the sense that the major element that links all lemmas (the only one which is not related directly to the RIM-decomposition is Lemma 3, but it is about the RIM-spinor itself), propositions, and theorems presented here is the pair of coefficients of the decomposition (or, in other words, the coordinates in the spinor-plane) of a given spinor in terms of RIM-spinors. Following this idea, one can study spinor properties in a very similar way if a given spinor is decomposable in terms of another. Thus, this work provides a working protocol that can be useful in other cases of the theory of spinors field of study.

With regard to direct physical applications, this work provides a homotopical method of construction of any possible spinor field allowed in the Spinor Theory of Gravity (STG), which is a theory of gravitation built via a class of solutions of the linearized Einstein equations of General Relativity constructed from RIM-spinors, ${ }^{17,19}$ i.e., a gravitation theory with RIM-spinors playing a fundamental role. Moreover, since bilinear covariants are associated with physical observables, we developed a way to easily verify the possible couplings of a particle associated with a given spinor in this theory, by means of their coefficients in the RIM-decomposition.

Considering the bijective linear map between Dirac and MDO spinors, one can think of its usefulness directly related to the task of understanding dark matter, which can be described by MDO fields. ${ }^{20}$ Once dark matter interacts very weakly with Standard Model (SM) particles, and aspects of Dirac fields are known in the SM context (in particular, the subset of RIM-decomposable Dirac spinors treated here), one can work with this Dirac-MDO mapping in further investigations on extending SM incorporating MDO spinors.

Further results concerning questions about more properties related to the homotopies in the spinor-plane are under investigation. Moreover, the behavior of MDO spinors and their bilinear covariants in this space is also a topic under study.

\section{ACKNOWLEDGMENTS}

The authors are grateful to Professor Julio Marny Hoff da Silva for useful conversation. D.B. would like to thank CAPES for the financial support, and R.J.B.R. would like to thank CAPES and CNPq (Grant No. 155675/2018-4) for the financial support. C.H.C.V. would like to thank CNPq (Grant No. 300381/2018-2) for the financial support.

\section{APPENDIX A: MASS-DIMENSION-ONE FIELDS AND THE FIERZ-PAULI-KOFINK IDENTITIES}

As can be seen in Ref. 9, it is possible to build the basis vectors for the mass-dimension-one spinor's case using the usual Clifford algebra. For any element $\Gamma$ belonging to such algebra, the FPK relation reads

$$
\left(\vec{\lambda}_{h} \Gamma \gamma_{\mu} \lambda_{h}\right)=\left(\vec{\lambda}_{h} \Gamma \lambda_{h}\right) \lambda_{h}-\left(\vec{\lambda}_{h} \Gamma \gamma_{5} \lambda_{h}\right) \gamma_{5} \lambda_{h}
$$

where $\Gamma \in\left\{\mathbb{1}, \gamma_{5}, \gamma_{\mu}, \Xi \gamma_{5} \gamma_{\mu} \Xi\right\}$. From the above relation, we obtain the following:

$$
J^{2}=A^{2}+B^{2}
$$

and we also have

$$
\begin{aligned}
\left(\vec{\lambda}_{h} \Xi \gamma_{5} \gamma_{\mu} \Xi \lambda_{h}\right) \gamma^{\mu} \lambda_{h} & =\left(\vec{\lambda}_{h} \Xi \gamma_{5} \Xi \lambda_{h}\right) \lambda_{h}-\left(\vec{\lambda}_{h} \Xi^{2} \lambda_{h}\right) \gamma_{5} \lambda_{h} \\
& =\left(\vec{\lambda}_{h} \gamma_{5} \lambda_{h}\right) \lambda_{h}-\left(\vec{\lambda}_{h} \lambda_{h}\right) \gamma_{5} \lambda_{h} .
\end{aligned}
$$


Note that

$$
\left[\Xi, \gamma_{5}\right]=0, \quad\left\{\gamma_{\mu}, \gamma_{5}\right\}=0, \quad \text { and } \quad \Xi^{2}=\mathbb{1}
$$

and with such relations at hands, one is able to write

$$
\begin{aligned}
\left(\overrightarrow{\lambda_{h}} \Xi \gamma_{5} \gamma_{\mu} \Xi \lambda_{h}\right) \gamma^{\mu} \gamma^{5} \lambda_{h} & =-\left(\overrightarrow{\lambda_{h}} \Xi \gamma_{5} \gamma_{\mu} \Xi \gamma_{5} \lambda_{h}\right) \gamma^{\mu} \lambda_{h}, \\
& =\left(\overrightarrow{\lambda_{h}} \Xi \gamma_{\mu} \Xi \lambda_{h}\right) \gamma^{\mu} \lambda_{h} .
\end{aligned}
$$

Finally, using relations (A3) and (A4), we obtain

$$
\begin{aligned}
J_{\mu} \gamma^{\mu} \lambda_{L} & =(A-i B) \lambda_{R}, \\
J_{\mu} \gamma^{\mu} \lambda_{R} & =(A+i B) \lambda_{L}, \\
K_{\mu} \gamma^{\mu} \lambda_{L} & =-(A-i B) \lambda_{R}, \\
K_{\mu} \gamma^{\mu} \lambda_{R} & =(A+i B) \lambda_{L} .
\end{aligned}
$$

\section{APPENDIX B: SOME COMMENTS ON THE LINEAR AND NON-LINEAR ASPECTS OF THE SPINOR FIELDS}

We know that the Dirac equation is linear with respect to the spinor fields

$$
\left(i \gamma^{\mu} \partial_{\mu}-M\right) \Psi^{D}=0,
$$

with $M$ being a mass parameter. The solutions $\Psi^{D}$ are called Dirac spinors, which, because of the linear aspect of the Dirac equation (B1), are said to be linear spinor fields.

The non-linear counterpart, which we call the Heisenberg equation, is given by

$$
\left[i \gamma^{\mu} \partial_{\mu}-2 s\left(A+i B \gamma^{5}\right)\right] \Psi^{H}=0,
$$

with $A:=\bar{\Psi}^{H} \Psi^{H}$ and $B:=i \bar{\Psi}^{H} \gamma^{5} \Psi^{H}$ being the bilinear covariants associated with the so-called Heisenberg spinor $\Psi^{H}$, and $s$ is a constant with dimension (length) ${ }^{2}$. The Heisenberg equation is non-linear with respect to the fields, as one can easily verify by rewriting it as

$$
i \gamma^{\mu} \partial_{\mu} \Psi^{H}-2 s \bar{\Psi}^{H} \Psi^{H} \Psi^{H}+2 s \bar{\Psi}^{H} \gamma^{5} \Psi^{H} \gamma^{5} \Psi^{H}=0 .
$$

Thus, Heisenberg spinors are general solutions of Equation (B2), and in this sense, we say that they are non-linear spinor fields.

Let us compare some aspects of the linear Dirac dynamics and the non-linear Heisenberg dynamics. For the linear case, a particular solution of the Dirac equation (plane waves) can be written as

$$
\partial_{\mu} \Psi^{D}=i k_{\mu} \Psi^{D}
$$

On the other hand, in the non-linear case of Heisenberg spinors $\Psi^{H}$, it is possible to find solutions ${ }^{2}$ defined by the property

$$
\partial_{\mu} \Psi^{H}=\left(a J_{\mu}+b K_{\mu} \gamma^{5}\right) \Psi^{H},
$$

with $a, b \in \mathbb{C}$ such that $2 s=i(a-b)$. Yet, the integrability condition forces us to have $\operatorname{Re}(a)=\operatorname{Re}(b)$. Now, a spinor $\Psi^{H}$ that satisfies condition (B5) is called a RIM spinor, with RIM standing for Restricted Inomata-McKinley.

The term "restricted" was created by Ref. 3 , and the reason is the following: in the original decomposition, ${ }^{1}$ the first term of the rhs of Eq. (B5) is given by $K^{\lambda} \gamma_{\lambda} \gamma_{\mu} \gamma^{5}$. The mapping between this term and $J_{\mu}$ is not something straightforward. In fact, it is given by means of a matrix operator that we will call G. For the construction of this matrix, we will begin introducing the so-called Inomata-McKinley spinors that are special Heisenberg spinors which satisfy

$$
\partial_{\mu} \Psi=\underbrace{\frac{1}{2} \epsilon}_{\tilde{a}}\left(\bar{\Psi} \gamma^{\lambda} \gamma_{5} \Psi\right) \gamma_{\lambda} \gamma_{\mu} \gamma_{5} \Psi \underbrace{-2 \epsilon}_{\tilde{b}}\left(\bar{\Psi} \gamma_{\mu} \gamma_{5} \Psi\right) \gamma_{5} \Psi .
$$

Then,

$$
\begin{aligned}
& \partial_{\mu} \Psi=\underbrace{\tilde{a}(-i)}_{a} K^{\lambda} \gamma_{\lambda} \gamma_{\mu} \gamma_{5} \Psi+\underbrace{\tilde{b}(-i)}_{b} K_{\mu} \gamma_{5} \Psi \Rightarrow \\
& \partial_{\mu} \Psi=a K^{\lambda} \gamma_{\lambda} \gamma_{\mu} \gamma_{5} \Psi+b K_{\mu} \gamma_{5} \Psi .
\end{aligned}
$$


Comparing with Eq. (B5), one wants that $K^{\lambda} \gamma_{\lambda} \gamma_{\mu} \gamma_{5} \Psi:=\varepsilon J_{\mu} \Psi$, implying that $K^{\lambda} \gamma_{\lambda} \gamma_{\mu} \gamma_{5}=\varepsilon J_{\mu} \rrbracket G$, for some operator $G$. Then, making $\varepsilon G \rightarrow G$, one has

$$
K^{\alpha} \gamma_{\alpha} \gamma_{\mu} \gamma_{5}=J_{\mu} G
$$

Multiplying by $J^{\mu}$ from the left, we obtain $J^{\mu} K^{\alpha} \gamma_{\alpha} \gamma_{\mu} \gamma_{5}=J^{2} G$, which leads us to

$$
G=\frac{1}{J^{2}}\left(J^{\mu} K^{\alpha} \gamma_{\alpha} \gamma_{\mu} \gamma_{5}\right)
$$

But $\left\{\gamma_{\nu}, \gamma_{\lambda}\right\}=\eta_{\nu \lambda}$, so we can write $\gamma_{\alpha} \gamma_{\mu}=\frac{1}{2}\left[\gamma_{\alpha}, \gamma_{\mu}\right]+\eta_{\mu \alpha}$. Thus,

$$
G=\frac{1}{J^{2}}\left(J^{\mu} K^{\alpha} \frac{1}{2}\left[\gamma_{\alpha}, \gamma_{\mu}\right]+J \cdot K^{0}\right) \gamma_{5}
$$

and finally, we have the explicit form for the operator that restricts the Inomata-McKinley spinors to the specific case of RIM spinors as follows:

$$
G=\frac{1}{2 J^{2}} J^{\mu} K^{\alpha}\left[\gamma_{\alpha}, \gamma_{\mu}\right] \gamma_{5}
$$

So, RIM spinors are special cases of Inomata-McKinley spinors, and both are particular cases of Heisenberg spinors. All of them are nonlinear fields since their dynamics are conducted by the Heisenberg equation. In a pictorial representation way, we can summarize this as $\Psi_{\text {Heisenberg }}^{H} \supset \Psi_{\text {Inomata-McKinley }}^{H} \supset \Psi_{\text {RIM }}^{H}$.

\section{APPENDIX C: SOME COMMENTS ON THE ELKO MASS-DIMENSION-ONE SPINOR FIELDS}

In the early days of mass dimension one spinors, the theory was presented in such a way that a breaking Lorentz term took part in the spin sums. As a net result, the associated quantum field was non-local and there was a preferred axis of symmetry. After all, the theory was shown to be invariant under $\operatorname{SIM}(2)$ and $\operatorname{HOM}(2)$ transformations, ${ }^{21}$ being then a typical theory carrying the Very Special Relativity symmetries. ${ }^{22}$ Quite recently, important advances on the spinor dual theory have opened the possibility of circumventing the Weinberg nogo theorem, proposing a spinor field of spin $1 / 2$ endowed with mass dimension one, local, and neutral with respect to gauge interactions, and whose theory respects Lorentz symmetries. ${ }^{5,23,24}$ We should bring to the scene the canonical Wigner work on the irreducible representations of the Poincaré group. ${ }^{25}$ By the Poincaré group, as usual, it is understood as the semi-simple extension of the orthochronous proper Lorentz group encompassing translations. By investigating the irreducible representations for this case, no particle as a fermion with canonical mass dimension one was found. ${ }^{26}$

Here, the situation is different, however, when discrete symmetries are taken into account, i.e., when not only the orthochronous proper group is considered. This point was also analyzed by Wigner, in a less known paper. ${ }^{27}$ Interestingly enough, Wigner found a fermionic irreducible representations whose behavior under $C, P$, and $T$ (where $C, P$, and $T$ stand for the charge conjugation, parity, and time reversal operators, respectively) are exactly what was expected for the bosonic fields; in other words, Wigner found (theoretically) a class of particles to be more specifically fermions, which are endowed with "bosonical" character. For concreteness, while conventional wisdom states that fermions belonging to the standard model (quarks and leptons) obey $T^{2}=-1\left((C P T)^{2}=-1\right)$ and bosons $T^{2}=+1\left((C P T)^{2}=+1\right)$, Wigner also has shown that, in the very realm of full Poincaré symmetries, it is also possible to have $T^{2}=+1$ for fermions $\left(\right.$ leading to $\left.(C P T)^{2}=+1\right)$. It turns out that the MDO field taken into account in this work performs a realization of the (indeed odd) aforementioned fermionic representation, from where we can adduce its "bosonical" character. ${ }^{26}$

In this way, what we want to emphasize is that through the analysis of Wigner's works, all arguments corroborate with what we already know about the characteristics of the MDO fermion in question. Although we are dealing with a fermion, it does not have the same mass dimensionality as the Dirac fermions do, in addition to the fact that it has the quantum field propagator similar to that of the scalar field and respect only the Klein-Gordon equation. That is, the MDO spinors are fermions carrying bosonical aspects.

Regarding its formal structure, the Elko spinors are defined as

$$
\lambda_{h}^{S / A}(\boldsymbol{p})=\left(\begin{array}{l} 
\pm i \Theta\left[\phi_{L}(\boldsymbol{p})\right]^{*} \\
\phi_{L}(\boldsymbol{p})
\end{array}\right),
$$

with $\Theta$ being the Wigner time-reversal operator, given by

$$
\Theta=\left(\begin{array}{rr}
0 & -1 \\
1 & 0
\end{array}\right) .
$$

Note that $\Theta\left[\phi_{L}(\boldsymbol{p})\right]^{*}$ and $\phi_{L}(\boldsymbol{p})$ are defined as right-hand and left-hand components (under Lorentz transformations), with the upper index $S / A$ standing for self-conjugated and anti-self-conjugated via charge conjugation operation

$$
\mathcal{C} \lambda_{h}^{S / A}= \pm \lambda_{h}^{S / A}
$$


while the lower index $h$ represents the helicity of each component. The dual helicity feature is encoded on the relations

$$
\vec{\sigma} \cdot \hat{p} \phi_{L}^{ \pm}(p)= \pm \phi_{L}^{ \pm}(p)
$$

and the other component has opposite helicity, i.e.,

$$
\vec{\sigma} \cdot \hat{p} \Theta\left[\phi_{L}^{ \pm}(p)\right]^{*}=\mp \Theta\left[\phi_{L}^{ \pm}(p)\right]^{*} .
$$

The helicity is simply flipped by the action of the Wigner time-reversal operator.

Referring to dynamics, such spinors do not fulfill the Dirac dynamical equation (B1) due to the fact that their representation spaces are not linked by the parity symmetry, but by the Wigner time-reversal operator. This way, MDO spinors dynamics are governed only by the Klein-Gordon equation. The last statement is translated into the mass dimensionality of the referred spinors.

\section{REFERENCES}

${ }^{1}$ A. Inomata and W. A. McKinley, “Geometric theory of neutrinos," Phys. Rev. Lett. 140(5B), B1467 (1965).

${ }^{2}$ M. Novello, “Constructing Dirac linear fermions in terms of non-linear Heisenberg spinors," Europhys. Lett. 80, 41001 (2007).

${ }^{3}$ D. Beghetto and J. M. Hoff da Silva, “The (restricted) Inomata-McKinley spinor representation and the underlying topology,” Europhys. Lett. 119, 40006 (2017).

${ }^{4}$ D. V. Ahluwalia-Khalilova and D. Grumiller, J. Cosmol. Astropart. Phys. 2005(07), 012.

${ }^{\mathbf{5}}$ D. V. Ahluwalia, “The theory of local mass dimension one fermions of spin one half,” Adv. Appl. Clifford Algebras 27, 2247 (2017).

${ }^{6}$ R. da Rocha and J. M. Hoff da Silva, “From Dirac spinor fields to eigenspinoren des ladungskonjugationsoperators,” J. Math. Phys. 48, 123517 (2007).

${ }^{7}$ J. M. Hoff da Silva and R. da Rocha, "From Dirac action to ELKO action," Int. J. Mod. Phys. A 24, 3227-3242 (2009).

${ }^{8}$ R. da Rocha and J. M. Hoff da Silva, "ELKO, flagpole and flag-dipole spinor fields, and the instanton hopf fibration," Adv. Appl. Clifford Algebras 20, 847 (2010).

${ }^{9}$ J. M. Hoff da Silva, C. H. Coronado Villalobos, R. J. Bueno Rogerio, and E. Scatena, “On the bilinear covariants associated to mass dimension one spinors,” Eur. Phys. J. 76, 563 (2016).

${ }^{10}$ W. E. Baylis, Clifford (Geometric) Algebras with Applications to Physics, Mathematics, and Engineering (Editorial Birkhäuser Boston, Cambridge, 1996).

${ }^{11}$ P. Lounesto, Clifford Algebra and Spinors, 2nd ed. (Cambridge University Press, Cambridge, 2001).

${ }^{12}$ R. da Rocha, L. Fabbri, J. M. Hoff da Silva, R. T. Cavalcanti and J. A. Silva-Neto, J. Math. Phys. 54, 102505 (2013).

${ }^{13}$ I. M. Benn and R. W. Tucker, An Introduction to Spinors and Geometry with Applications in Physics (Adam Hilger, Bristol, 1987).

${ }^{14}$ E. Majorana, “Atomi orientati in campo magnetico variabile," Il Nuovo Cimento 9, 43 (1932).

${ }^{15}$ C. H. Coronado Villalobos and R. J. Bueno Rogerio, “The connection between Dirac dynamic and parity symmetry," Europhys. Lett. 116, 60007 (2016).

${ }^{16}$ W. Heisenberg, "Research on the non-linear spinor theory with indefinite metric in hilbert space," in Scientific Review Papers, Talks, and Books Wissenschaftliche Übersichtsartikel, Vorträge und Bücher, Gesammelte Werke/Collected Works, edited by W. Blum, H. P. Dürr, and H. Rechenberg (Springer, Berlin, Heidelberg, 1984), Vol. B.

${ }^{17}$ S. Joffily and M. Novello, Gen. Relativ. Gravitation 48, 151 (2016).

${ }^{18}$ W. Heisenberg, “Quantum theory of fields and elementary particles," Rev. Mod. Phys. 29(3), 269 (1957).

${ }^{19}$ M. Novello, "A spinor theory of gravity and the cosmological framework," J. Cosmol. Astropart. Phys. 2007(06), 018.

${ }^{20}$ D. V. Ahluwalia-Khalilova, “Dark matter, and its darkness,” Int. J. Mod. Phys. D 15(12), 2267-2278 (2006).

${ }^{21}$ D. V. Ahluwalia and S. P. Horvath, "Very special relativity as relativity of dark matter: The Elko connection," J. High Energy Phys. 2010(11), 078.

${ }^{22}$ A. G. Cohen and S. L. Glashow, "Very special relativity," Phys. Rev. Lett. 97, 021601 (2006).

${ }^{23}$ D. V. Ahluwalia, “Evading Weinberg's no-go theorem to construct mass dimension one fermions: Constructing darkness,” Europhys. Lett. 118, 60001 (2017).

${ }^{24}$ R. J. Bueno Rogerio and J. M. Hoff da Silva, “The local vicinity of spins sum for certain mass dimension one spinors,” Europhys. Lett. 118, 10003 (2017).

${ }^{25}$ E. P. Wigner, “On unitary representations of the inhomogeneous Lorentz group," Ann. Math. 40, 149 (1939).

${ }^{26}$ R. J. Bueno Rogerio, J. M. Hoff da Silva, M. Dias, and S. H. Pereira, “Effective Lagrangian for a mass dimension one fermionic field in curved spacetime," J. High Energy Phys. 2018(2), 145.

${ }^{27}$ E. P. Wigner, "Unitary representations of the inhomogeneous Lorentz group including reflections," in Group Theoretical Concepts and Methods in Elementary Particle Physics, Lectures of the Istanbul Summer School of Theoretical Physics, 1962, edited by F. Gürsey (Gordon \& Breach, New York, 1964), pp. 37-80.

${ }^{28}$ In this work, what we call by a manifold with underlying trivial topology is a manifold $M$ that has a trivial fundamental group $\pi_{1}(M)=0$. Otherwise, the manifold $M$ will be said to have a non-trivial topology.

${ }^{29}$ The fundamental field equations must be non-linear in order to represent interaction. The masses of the particles should be a consequence of this interaction. ${ }^{18}$

${ }^{30}$ The authors choose to work in abstract only with the $\lambda_{h}^{S}$ spinors since the physical content holds the same for all the other MDO spinors, one differing from the other only by a constant phase. 